\title{
Arm Swing Magnitude and Asymmetry During Gait in the Early Stages of Parkinson's Disease
}

\author{
Michael D. Lewek, PT, PhD ${ }^{1}$, Roxanne Poole, BS ${ }^{2}$, Julia Johnson, MD $^{2}$, Omar Halawa, BS ${ }^{2}$, \\ and Xuemei Huang, MD, $\mathrm{PhD}^{2,3}$ \\ 1 Department of Allied Health Sciences, Division of Physical Therapy, University of North Carolina, \\ Chapel Hill, NC 27599-7135 \\ 2 Department of Neurology, University of North Carolina, Chapel Hill, NC 27599-7125 \\ ${ }^{3}$ Department of Neurology, Penn State University-Milton S. Hershey Medical Center, Hershey, PA \\ 17033-H037
}

\begin{abstract}
The later stages of Parkinson's disease (PD) are characterized by altered gait patterns. Although decreased arm swing during gait is the most frequently reported motor dysfunction in individuals with PD, quantitative descriptions of gait in early PD have largely ignored upper extremity movements. This study was designed to perform a quantitative analysis of arm swing magnitude and asymmetry that might be useful in the assessment of early PD. Twelve individuals with early PD (in "off" state) and eight controls underwent gait analysis using an optically-based motion capture system. Participants were instructed to walk at normal and fast velocities, and then on heels (to minimize push-off). Arm swing was measured as the excursion of the wrist with respect to the pelvis. Arm swing magnitude for each arm, and inter-arm asymmetry, were compared between groups. Both groups had comparable gait velocities ( $\mathrm{p}=0.61$ ), and there was no significant difference between the groups in the magnitude of arm swing in all walking conditions for the arm that swung more $(p=0.907)$ or less $(\mathrm{p}=0.080)$. Strikingly, the PD group showed significantly greater arm swing asymmetry (asymmetry angle: $13.9 \pm 7.9 \%$ ) compared to the control group (asymmetry angle: $5.1 \pm 4.0 \%$; $\mathrm{p}=0.003$ ). Unlike arm swing magnitude, arm swing asymmetry unequivocally differs between people with early PD and controls. Such quantitative evaluation of arm swing, especially its asymmetry, may have utility for early and differential diagnosis, and for tracking disease progression in patients with later PD.
\end{abstract}

\section{Keywords}

Parkinson's Disease; gait; biomechanics; arm swing; arm swing asymmetry

\footnotetext{
(C) 2009 Elsevier B.V. All rights reserved.
}

Corresponding Author: Xuemei Huang MD, PhD Penn State University-Milton S. Hershey Medical Center, H037 Department of Neurology, 500 University Drive Hershey, PA 17033-0850 Phone: 717-531-1803 Fax: 717-531-0465 xuemei@ psu.edu.

Publisher's Disclaimer: This is a PDF file of an unedited manuscript that has been accepted for publication. As a service to our customers we are providing this early version of the manuscript. The manuscript will undergo copyediting, typesetting, and review of the resulting proof before it is published in its final citable form. Please note that during the production process errors may be discovered which could affect the content, and all legal disclaimers that apply to the journal pertain.

Conflict of interest statement: The authors have nothing to disclose related to any financial and personal relationships with other people or organizations that could inappropriately influence (bias) our work. 
Parkinson's disease (PD) is the second most common age-related, neurodegenerative disorder. Tremor, rigidity, bradykinesia, and postural instability are hallmarks for the diagnosis of PD. ${ }^{1}$ Abnormal gait (i.e., small "shuffling" steps) is common in the later stages of PD, and can be characterized by reduced walking velocity, stride length, swing/stance time ratio, and cadence. $2-4$

There are, however, few comprehensive studies that describe the changes in upper extremity motions despite the fact that decreased arm swing is the most frequently reported motor dysfunction in individuals with PD. ${ }^{5}$ Additionally, a reduction in arm swing has been reported to be associated with an increased risk of falls for patients with PD. ${ }^{6}$ Previous attempts to quantify arm swing in patients with PD were focused solely on sagittal plane shoulder kinematics, $4^{7} 7^{-} 9$ yet the total amount of arm swing during walking incorporates both elbow ${ }^{10}$ and trunk kinematics. This suggests the importance of examining the trajectory of the end effector (e.g., wrist/hand) when quantifying arm swing.

The asymmetric process of nigrostriatal dopaminergic denervation occurring in PD contributes to an asymmetrical presentation of motor dysfunctions in PD. ${ }^{11,12}$ The presence of motor asymmetry may be helpful for increasing the accuracy of PD diagnosis. ${ }^{13}$ Although lower extremity asymmetry during gait has been well quantified in individuals with PD, $4,14-16$ the arm swing asymmetry during walking has only been described qualitatively. ${ }^{1,6,17}$ For this reason, the present work focused on arm swing magnitude, and its side-to-side asymmetry, during gait in individuals with early PD. Based on previous descriptions of altered shoulder motion, ${ }^{4,7-9}$ and the asymmetric onset of motor symptoms, 11,12 we hypothesized that arm swing in individuals with early PD would be decreased in amplitude and more asymmetric when compared to controls. In designing experiments to measure arm swing in individuals with PD, we were aware of a phenomenon known as "kinesia paradoxica". ${ }^{18}$ In particular, individuals with PD may show dysfunction in a given task, such as gait, yet perform very well when challenged or when the task is externally cued. Participants therefore walked (1) at their normal velocity, (2) as fast as they could (a challenge condition), and (3) on their heels (an unnatural gait in which individuals maximized stride length while only letting their heels touch the ground).

\section{Methods}

\section{Participants}

Twelve individuals within three years of PD diagnosis and eight control participants were tested (Table 1). PD diagnosis was made by a movement disorder specialist according to published guidelines.1 All participants in the PD group were treated with dopaminergic replacements and showed a dramatic clinical improvement. Individuals with PD were tapered off all antiparkinsonian drugs at least 12 hours prior to testing. A group consensus previously recommended that a practically defined "off" state be operationally defined as a patient's condition after not receiving anti-parkinsonian medication for 12 hours. 19 All participants were examined by a movement disorder physician (XH or JJ), and were free of muscular weakness, history of stroke, pathology or surgery to the upper extremities, or major medical illness. All individuals performed the Berg Balance scale prior to testing to estimate fall risk. A cutoff of 54 has recently been proposed to predict risk of falling in patients with PD. ${ }^{20}$ The study protocol was reviewed and approved by the Institutional Review Board (IRB \# 07-2070). Written informed consent was obtained from all participants prior to participation.

\section{Gait Analysis}

All participants underwent a single gait analysis session to determine the motion of the arms, trunk, pelvis and lower extremities. Body segments were tracked during gait using an eight 
camera, passive, three-dimensional motion analysis system (Vicon/Peak, Lake Forest, CA) sampling at $120 \mathrm{~Hz}$. Heel strikes were obtained from foot switches (Motion Lab Systems, Baton Rouge, LA) synchronized with the Peak system for simultaneous collection at $1080 \mathrm{~Hz}$. Retroreflective markers were placed bilaterally on the lateral aspect of the fifth metatarsal head, medial and lateral malleoli, medial and lateral femoral condyles, greater trochanters, iliac crests, acromion processes, spinous process of $\mathrm{C} 7$, medial and lateral humeral condyles, and the styloid process of the ulna and radius to indicate the ends of the segments and to identify appropriate joint centers. Rigid thermoplastic shells, each with four markers firmly affixed, were attached to the posterolateral aspect of the thighs and shanks and covered with an elastic wrap to minimize movement. Marker triads were placed on the sacrum and both feet. Prior to the collection of walking trials, a static standing trial was recorded to identify joint centers with respect to each segment coordinate system.

Marker position and foot switch data were recorded simultaneously while participants walked across a 25-foot walkway under three conditions: 1) normal (e.g., self-selected) walking velocity ("Normal"), 2) fastest possible walking velocity while maintaining safety and without jogging ("Fast"), and 3) walking on the heels at a self-selected velocity ("Heel-Walking"). Individuals repeated each condition five times. For Heel-Walking, participants were instructed to maximize stride length while only letting the heels touch the ground (e.g., toes up). The intention of the Heel-Walking condition was to minimize push-off, and thus accentuate arm swing to generate propulsive forces. No instructions or feedback regarding arm swing were provided for any condition.

\section{Data Management and Processing}

Data analysis software (Peak Motus) was used to identify the locations of the markers in the lab coordinate system, and to low-pass filter these marker trajectories at $6 \mathrm{~Hz}$. The markers defined a kinematic model for tracking the three-dimensional motion of the arms, trunk, pelvis, and lower limb segments. All segment coordinate systems were defined with the positive Xaxis to the right, positive $\mathrm{Y}$-axis facing anteriorly, and positive $\mathrm{Z}$-axis pointing superiorly. Visual3D software (C-Motion, Germantown, MD) estimated segment properties from measured anthropometric values. ${ }^{21}$ All segments were modeled as a frustra of right cones, except for the pelvis and the trunk that were each modeled as cylinders.

Our primary interest was in quantifying arm swing: we defined this operationally as the distance traveled by the wrist in the anterior/posterior and medial/lateral directions with respect to the pelvis within a stride (see Figure 1). To account for pelvic rotations during walking, and movement of the participant through the lab space, we expressed the location of the distal end of the forearm segment (wrist joint) in the sagittal and frontal planes of the pelvis's local coordinate system. The arm swing for each arm was time-normalized to $100 \%$ of the gait cycle, and an ensemble average was calculated. The total distance traveled then was calculated for each arm to obtain a representative value for arm swing.

The arm that swung less (ArmSwing less $_{\text {) }}$ ) and the arm that swung more (ArmSwing analyzed separately for each group. Separating arm swing in this way allowed for the calculation of arm swing asymmetry (ASA) using the symmetry angle described by Zifchock and colleagues. ${ }^{22}$

$$
\operatorname{ASA}=\frac{\left(45^{\circ}-\arctan \left(\text { ArmSwing }_{\text {more }} / \text { ArmS wing }_{\text {less }}\right)\right)}{90^{\circ}} \times 100 \%
$$

The ASA is designed to represent asymmetry in arm swing magnitude between each arm. A value of 0.00 would indicate that both arms are moving exactly the same magnitude. 
To ensure that trunk rotation did not influence arm swing, we quantified trunk rotation to the left and to the right as the transverse plane angular rotation of the thorax with respect to the pelvic coordinate system. The sequence of rotations for this calculation was Z-X-Y (axial rotation, flexion/extension, sidebending). The magnitude of trunk rotation was quantified as the total side-to-side rotation of the thorax during a stride cycle. We then calculated the trunk rotation asymmetry (TRA) as follows:

$$
\mathrm{TRA}=\frac{\left(45^{\circ}-\arctan \left(\text { TrunkRotation }_{\text {more }} / \text { TrunkRotation }_{\text {less }}\right)\right)}{90^{\circ}} \times 100 \%
$$

In addition to arm and trunk movements, we calculated gait velocity as the velocity of the pelvic markers through the lab coordinate system in the direction of forward progress. Asymmetry of the lower limbs was defined as the asymmetry in stride time. The time, in seconds, from heel strike to subsequent heel strike was calculated for both sides. The stride time asymmetry (STA) was then calculated as follows:

$$
\mathrm{STA}=\frac{\left(45^{\circ}-\arctan \left(\text { StrideTime }_{\text {more }} / \text { StrideTime }_{\text {less }}\right)\right)}{90^{\circ}} \times 100 \%
$$

\section{Statistical Analysis}

Statistical analysis was performed using SPSS (version 15.0, Chicago, IL). Outcome variables of interest included arm swing magnitude of the arm that swung more (ArmSwing ${ }_{\text {more }}$ ) and less (ArmSwing less $_{\text {, }}$, trunk rotation, upper and lower extremity and trunk rotation asymmetry (e.g., ASA, STA, and TRA), and gait velocity. Assessments were made in both the PD and control groups during each of the three gait conditions (Normal, Fast, and Heel-Walking).

Variables were compared between groups using two-way repeated measures ANOVA (group $\times$ condition) repeated for condition. Post-hoc testing using Bonferroni correction for multiple comparisons was used to evaluate significant main effects. A threshold for determining arm swing asymmetry during Normal walking was calculated as two standard deviations above the mean of ASA for the control group. Using the calculated threshold, we categorized individuals as either high or low asymmetry, and used a Fisher's Exact test to compare the distribution between groups (control vs. PD). For all statistical analyses an $\alpha=0.05$ was used to establish statistical significance.

\section{Results}

Gait velocities were comparable between groups for all conditions $(\mathrm{F}(1,18)=0.01 ; \mathrm{p}=0.922)$, although gait velocities were significantly different among walking conditions $(F(2,36)=$ 76.68; $\mathrm{p}<0.001$ ) (Table 2). Specifically, both the PD and control groups had a higher gait velocity during the Fast condition compared to the Normal or Heel-Walking conditions $(\mathrm{p}<0.001)$. No difference was seen between the Normal and Heel-Walking conditions $(\mathrm{p}=0.156)$.

Across walking conditions, Arm Swing more $(\mathrm{F}(1,18)=0.014 ; \mathrm{p}=0.907)$ and Arm Swing less $_{\text {( }}(\mathrm{F}$ $(1,18)=3.447 ; \mathrm{p}=0.080)$ were not significantly different between the PD and control groups (Table 2). Conversely, arm swing was affected significantly by walking condition (Arm Swing $_{\text {more }}: F(2,36)=88.271: p<0.001$; Arm Swing less $\left.: F(2,36)=53.150: p<0.001\right)$. Fast walking produced significantly greater arm swing of both arms than Normal walking $(p<0.001)$, whereas Heel-Walking generated greater arm swing than both the $\operatorname{Normal}(\mathrm{p}<0.001)$ and Fast $(\mathrm{p}<0.001)$ walking conditions. 
The PD group exhibited significantly greater ASA compared to the control group (Figure 2) across all walking conditions $(F(1,18)=11.890 ; p=0.003)$. Post-hoc testing revealed that ASA measures were significantly greater in the PD group for the Normal $(\mathrm{p}<0.001)$, and Fast $(\mathrm{p}=0.015)$ conditions, but not for Heel-Walking $(\mathrm{p}=0.062)$.

No significant main effect of walking condition was observed for ASA $(\mathrm{F}(2,36)=0.059$; $\mathrm{p}=0.943$ ), and there was no significant interaction effect (group $\times$ condition: $F(2,36)=2.493$; $\mathrm{p}=0.097$ ). We established a threshold of excessive asymmetry during Normal walking based on the values from the control group (threshold $=7.4 \%$ ). Based on this threshold, 10 of $12(83 \%)$ participants in the PD group and 0 of $8(0 \%)$ of the control group had excessive ASA during normal walking $(\mathrm{p}<0.001)$.

The magnitude of trunk rotation (Table 2) was not significantly different between the PD and control groups $(F(1,18)=0.386 ; p=0.542)$. A main effect for condition $(F(2,36)=50.320$; $\mathrm{p}<0.001)$ was noted, such that Fast walking produced significantly greater trunk rotation than Normal walking $(\mathrm{p}<0.001)$, and Heel-Walking generated greater trunk rotation than both the Normal $(p<0.001)$ and Fast $(p<0.001)$ walking conditions. Our purpose for measuring trunk rotation was to ensure that ASA values were not simply a reflection of asymmetrical trunk rotation. Importantly, we observed that TRA (Table 2) was not significantly different between the PD and control groups $(\mathrm{F}(1,18)=3.371 ; \mathrm{p}=0.083)$. A significant main effect of condition was observed for TRA $(\mathrm{F}(2,36)=3.564 ; \mathrm{p}=0.039)$. Post-hoc tests indicated that TRA was greater during Heel-Walking compared to the Normal speed condition $(\mathrm{p}=0.040)$.

The STA (Table 2) was not significantly different between groups $(\mathrm{F}(1,18)=1.325 ; \mathrm{p}=0.265)$. Furthermore, STA was not significantly different among walking conditions $(\mathrm{F}(2,36)=1.682$; $\mathrm{p}=0.200$ ), and there was no significant interaction effect (group $\times$ condition: $F(2,36)=0.304$; $\mathrm{p}=0.740$ ).

\section{Discussion}

We believe that the current study is the first comprehensive study of arm swing in patients with early PD, and suggests that asymmetry, but not the magnitude of arm swing, may be an early sign of the disease. This suggests that a quantitative description of arm swing, especially its asymmetry, should be evaluated in future studies because of the potential implication for early and differential diagnosis, and for predicting and tracking PD progression.

It was somewhat unexpected that no significant differences in arm swing magnitude were found between PD and control groups because decreased arm swing has been described as an early sign of PD. ${ }^{23}$ There are several possible factors influencing our finding, including the fact that all participants with PD were in the early stage of their disease, may still have been influenced by residual drug effects that had not "washed out", and/or performed better because they were aware that they were being observed.

Most notably, these data show an unequivocal distinction in arm swing asymmetry between individuals with early PD and controls during Normal walking. The asymmetric process of nigrostriatal dopaminergic denervation and motor asymmetry occurring in PD has been welldescribed.11,12 The clinically-judged presence of motor asymmetry (tremor, rigidity, and bradykinesia) has been used to help increase the accuracy of PD diagnosis. ${ }^{13}$ The current data now provide quantitative measurements for one aspect of this motor asymmetry.

Although others have reported the presence of swing time asymmetry in the lower extremities of individuals with de novo PD16 the current data showed asymmetry only in arm, not leg, swing in individuals with early PD. This discrepancy may be due to differences in the methodology which was employed. First, we used a novel asymmetry angle purported to 
correct for artificially inflated values of previous asymmetry measures. ${ }^{22}$ Moreover, all individuals in our PD group were treated with anti-parkinsonian medication, whereas prior studies used drug naïve patients. Although we attempted to wash out drug effects overnight (12 hours), there will be residual medication benefit that could have masked the STA. Nevertheless, the current data suggest that arm swing asymmetry may either precede, or be more robust than, lower extremity asymmetry in marking the early stages of PD.

Similar to prior reports, we demonstrated that arm swing magnitude is associated with gait velocity in patients with $\mathrm{PD}^{7}$ and controls. ${ }^{10}, 26,27$ Thus, findings that there is decreased arm swing in individuals in the later stages of PD may be associated with the diminished gait velocity commonly observed with PD. $4,{ }^{7}$ It is interesting to note that whereas arm swing magnitude was significantly modified by both walking velocity (Normal or Fast) and pattern (natural or unnatural), arm swing asymmetry was relatively resistant to the changes in walking conditions. We therefore propose that arm swing asymmetry may serve as a more reliable parameter than arm swing magnitude in studying PD.

Because reductions in trunk rotation during gait are well known to be present in individuals with $\mathrm{PD},{ }^{4,26}$ it is possible that the arm swing measurements could have been confounded by alterations in trunk rotation between groups. The fact that trunk rotation asymmetry was not different between the groups in our study suggests that the arm swing asymmetry measurement was able to account for any discrepancies in trunk rotation.

There has been a great deal of effort to detect PD in preclinical states using a variety of nonmotor symptoms (such as olfaction, autonomic dysfunction and sleep disorders). ${ }^{23}, 28$ Although such non-motor symptoms may be sensitive for the early detection of PD, they remain non-specific.28 Arm swing belongs to the domain of motor function, and the dramatic differences in arm swing asymmetry between early PD and control groups suggests that arm swing asymmetry may have unique use in the early and differential diagnosis of PD, and indeed, in monitoring its progression.

\section{Acknowledgments}

This work was supported in part by the National Institutes of Health [R01NS06722 and K23AG21491 to XH, and UL1RR025747 from the Clinical and Translational Science Award program of the Division of Research Resources]; and the UNC Center for Human Movement Sciences. We thank Richard Mailman for his insightful feedback about the manuscript.

\section{References}

1. Gelb DJ, Oliver E, Gilman S. Diagnostic criteria for parkinson disease. Arch Neurol 1999;56(1):3339. [PubMed: 9923759]

2. Alice N, Fabienne C, Anne-Marie W, Kaat D. Does freezing in parkinson's disease change limb coordination? A kinematic analysis. J Neurol 2007;254(9):1268-1277. [PubMed: 17401738]

3. Ferrarin M, Rizzone M, Bergamasco B, Lanotte M, Recalcati M, Pedotti A, Lopiano L. Effects of bilateral subthalamic stimulation on gait kinematics and kinetics in parkinson's disease. Exp Brain Res 2005;160(4):517-527. [PubMed: 15502989]

4. Knutsson E. An analysis of parkinsonian gait. Brain 1972;95(3):475-486. [PubMed: 4655275]

5. Nieuwboer A, De Weerdt W, Dom R, Lesaffre E. A frequency and correlation analysis of motor deficits in parkinson patients. Disabil Rehabil 1998;20(4):142-150. [PubMed: 9571381]

6. Wood BH, Bilclough JA, Bowron A, Walker RW. Incidence and prediction of falls in parkinson's disease: A prospective multidisciplinary study. J Neurol Neurosurg Psychiatry 2002;72(6):721-725. [PubMed: 12023412] 
7. Carpinella I, Crenna P, Marzegan A, Rabuffetti M, Rizzone M, Lopiano L, Ferrarin M. Effect of 1dopa and subthalamic nucleus stimulation on arm and leg swing during gait in parkinson's disease. Conf Proc IEEE Eng Med Biol Soc 2007:6665-6668. [PubMed: 18003554]

8. Zijlmans JC, Poels PJ, Duysens J, van der Straaten J, Thien T, van't Hof MA, Thijssen HO, Horstink MW. Quantitative gait analysis in patients with vascular parkinsonism. Mov Disord 1996;11(5):501508. [PubMed: 8866491]

9. Behrman AL, Teitelbaum P, Cauraugh JH. Verbal instructional sets to normalise the temporal and spatial gait variables in parkinson's disease. J Neurol Neurosurg Psychiatry 1998;65(4):580-582. [PubMed: 9771792]

10. Kuhtz-Buschbeck JP, Brockmann K, Gilster R, Koch A, Stolze H. Asymmetry of arm-swing not related to handedness. Gait Posture 2008;27(3):447-454. [PubMed: 17616462]

11. Yust-Katz S, Tesler D, Treves TA, Melamed E, Djaldetti R. Handedness as a predictor of side of onset of parkinson's disease. Parkinsonism Relat Disord. 2008

12. Uitti RJ, Baba Y, Whaley NR, Wszolek ZK, Putzke JD. Parkinson disease: Handedness predicts asymmetry. Neurology 2005;64(11):1925-1930. [PubMed: 15955945]

13. Hughes AJ, Ben-Shlomo Y, Daniel SE, Lees AJ. What features improve the accuracy of clinical diagnosis in parkinson's disease: A clinicopathologic study. Neurology 1992;42(6):1142-1146. [PubMed: 1603339]

14. Plotnik M, Giladi N, Balash Y, Peretz C, Hausdorff JM. Is freezing of gait in parkinson's disease related to asymmetric motor function? Ann Neurol 2005;57(5):656-663. [PubMed: 15852404]

15. Yogev G, Plotnik M, Peretz C, Giladi N, Hausdorff JM. Gait asymmetry in patients with parkinson's disease and elderly fallers: When does the bilateral coordination of gait require attention? Exp Brain Res 2007;177(3):336-346. [PubMed: 16972073]

16. Baltadjieva R, Giladi N, Gruendlinger L, Peretz C, Hausdorff JM. Marked alterations in the gait timing and rhythmicity of patients with de novo parkinson's disease. Eur J Neurosci 2006;24(6): 1815-1820. [PubMed: 17004944]

17. Lee PH, Joo US, Yong SW, Huh K. Asymmetric freezing of gait in hemiparkinsonismhemiatrophy. Neurology 2004;63(2):E7. [PubMed: 15277660]

18. Souques AA. Older description of parkinsonian persons who can run much easier than walk. Rev Neurol (Paris) 1921;37:559-560.

19. Langston JW, Widner H, Goetz CG, Brooks D, Fahn S, Freeman T, Watts R. Core assessment program for intracerebral transplantations (capit). Mov Disord 1992;7(1):2-13. [PubMed: 1557062]

20. Dibble LE, Lange M. Predicting falls in individuals with parkinson disease: A reconsideration of clinical balance measures. J Neurol Phys Ther 2006;30(2):60-67. [PubMed: 16796770]

21. Dempster WT. Space requirements of the seated operator: Geometrical, kinematic, and mechanical aspects of the body with special reference to the limbs. WADC technical report 1955:55-159.

22. Zifchock RA, Davis I, Higginson J, Royer T. The symmetry angle: A novel, robust method of quantifying asymmetry. Gait Posture 2008;27(4):622-627. [PubMed: 17913499]

23. Becker G, Muller A, Braune S, Buttner T, Benecke R, Greulich W, Klein W, Mark G, Rieke J, Thumler R. Early diagnosis of parkinson's disease. J Neurol 2002;249(Suppl 3):III, 40-48. [PubMed: 12522572]

24. Wagenaar RC, van Emmerik RE. Resonant frequencies of arms and legs identify different walking patterns. J Biomech 2000;33(7):853-861. [PubMed: 10831760]

25. Donker SF, Mulder T, Nienhuis B, Duysens J. Adaptations in arm movements for added mass to wrist or ankle during walking. Exp Brain Res 2002;146(1):26-31. [PubMed: 12192574]

26. Murray MP, Sepic SB, Barnard EJ. Patterns of sagittal rotation of the upper limbs in walking. Phys Ther 1967;47(4):272-284. [PubMed: 6040248]

27. Kubo M, Wagenaar RC, Saltzman E, Holt KG. Biomechanical mechanism for transitions in phase and frequency of arm and leg swing during walking. Biol Cybern 2004;91(2):91-98. [PubMed: 15351887]

28. Siderowf A, Stern MB. Premotor parkinson's disease: Clinical features, detection, and prospects for treatment. Ann Neurol 2008;64(Suppl 2):S139-147. [PubMed: 19127581] 
29. Doty RL. The olfactory system and its disorders. Semin Neurol 2009;29(1):74-81. [PubMed: 19214935] 


\section{Determination of Arm Swing Magnitude}
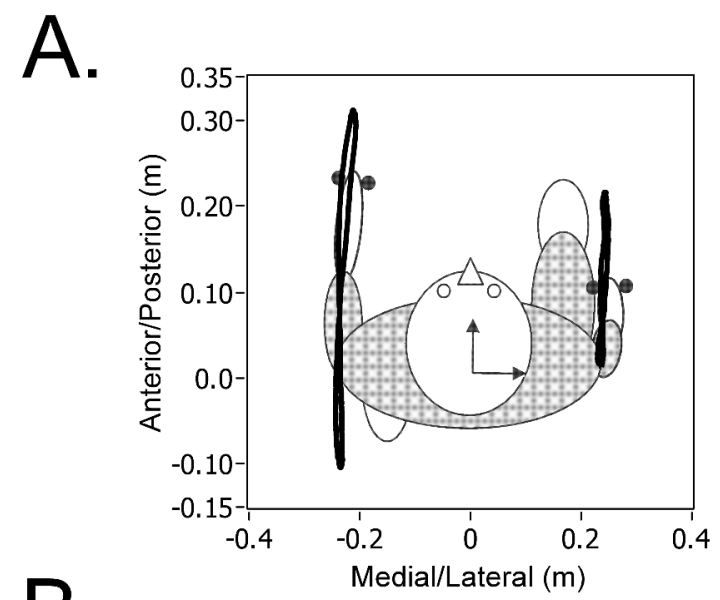

B.

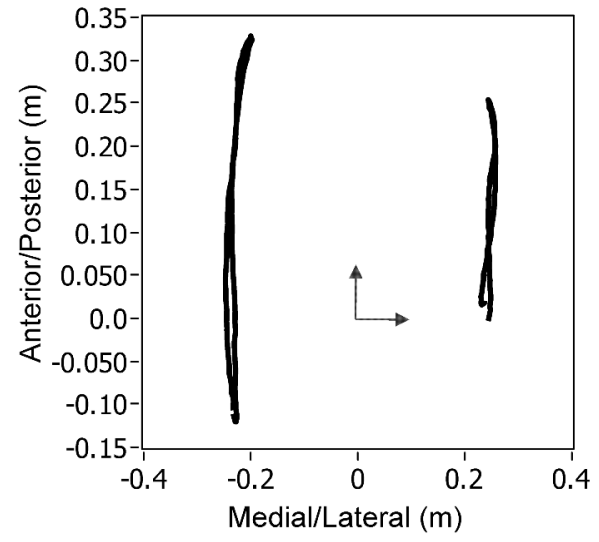

C.

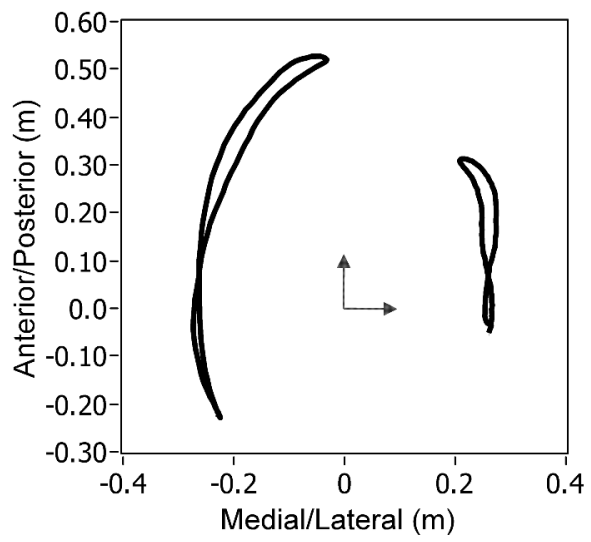

Figure 1.

Representative example of arm swing for an individual from PD group for an entire stride during (A) Normal, (B) Fast, and (C) Heel-Walking conditions. Arm swing magnitude was calculated as the total distance that the wrist travelled during an entire stride (e.g., path depicted by thick black lines), with respect to the origin of the pelvis (shown at 0,0 in the figures). 

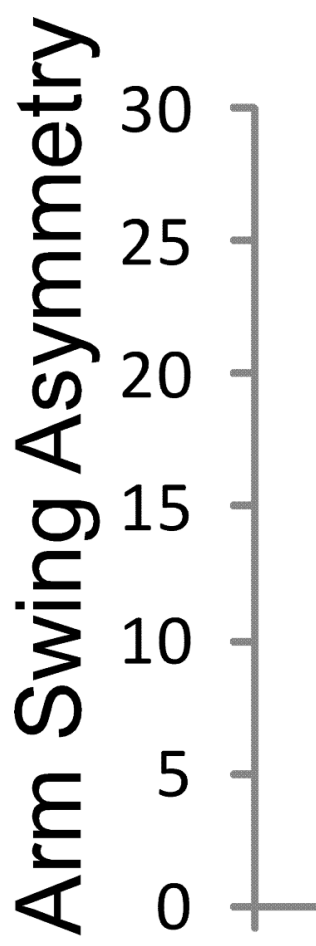

$\square$ PD Control
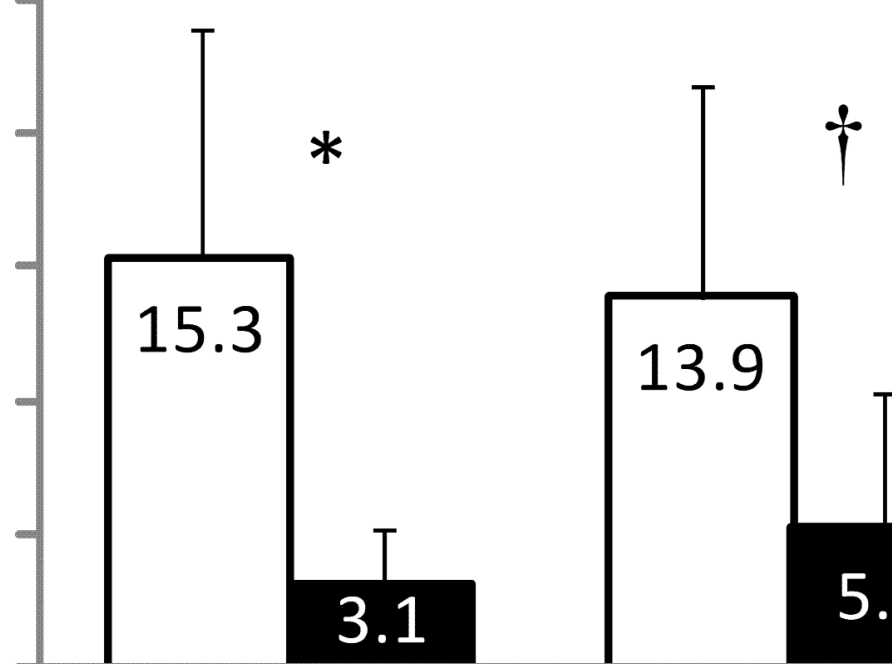

Normal

Fast

\section{Heel}

Figure 2.

Arm swing asymmetry for the Normal, Fast, and Heel-Walking conditions is shown for the PD (white bars) and control (black bars) groups. Values represent mean and standard deviations. *: $\mathrm{p}<0.001, \dagger: \mathrm{p}<0.05$ 
Table 1

Participant's Demographic Information

\begin{tabular}{llll}
\hline & PD group & control group & \\
\hline Age & $68 \pm 8$ years & $61 \pm 12$ years & $\mathrm{p}=0.148$ \\
Gender & 9 Males/3 Females & 5 Males/ 3 Females & $\mathrm{p}=0.642$ \\
More affected side & 10 Right/ 2 Left & N/A & p=0.999 \\
Berg balance Scale & $54 \pm 2$ & N/A & \\
Handedness & 11 Right/ 1 Left & 8 Right/ 0 Left & N/A \\
Months of PD Diagnosis & $24 \pm 10$ months & N/A & N/A \\
H\&Y score at "off" drug stage & $1.29 \pm 0.40$ & $11.25 \pm 5.55$ &
\end{tabular}




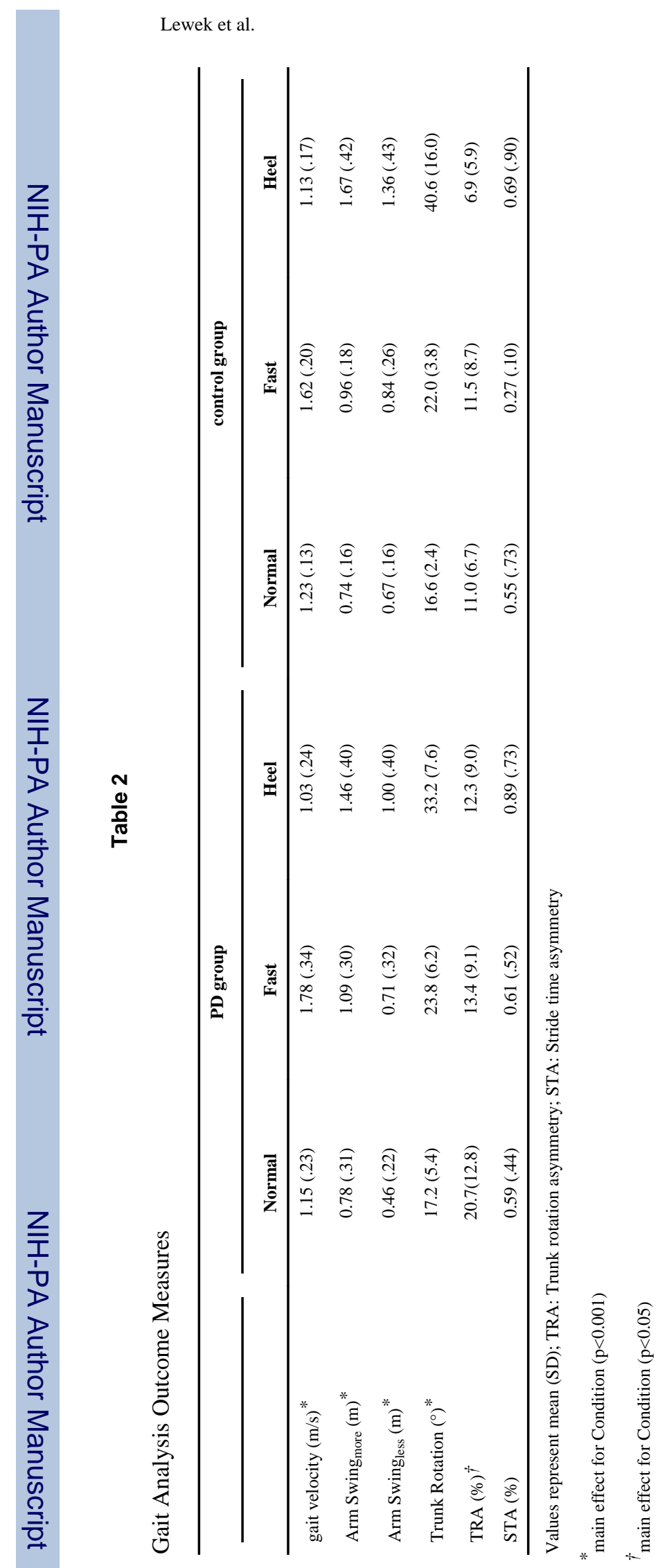

Gait Posture. Author manuscript; available in PMC 2011 February 1. 